\title{
Laparoscopic Ureterolithotomy Versus Ureteroscopy In Management Of Upper Ureteral Stone Larger Than 15 Mm In Adults
}

\author{
Mohamed Zaki Ali, Ahmed Mohammed Saafan, Magdy Mostafa Saleh, Mostafa Ahmed \\ Mostafa ElBakry* \\ Departments of Urology, Faculty of Medicine, Aswan University \\ *Corresponding author: Mostafa Ahmed Mostafa ElBakry, Mobile: 01003706454, Email: urobakry@gmail.com
}

\begin{abstract}
Background: There are multiple approaches for treatment of patients with upper ureteral stones. Extracorporeal shockwave lithotripsy (SWL), ureterorenoscopy (URS), percutaneous nephrolithotomy (PCNL), laparoscopic ureterolithotomy (LU) and open ureterolithotomy each has advantages and disadvantages. Although SWL is minimally invasive and can be performed as an outpatient procedure, disadvantages include a high retreatment rate, long treatment time, and inability to dissect a large or impacted stone. Objective: The aim of this study was to compare between laparoscopic ureterolithotomy and retrograde ureteroscopy for treatment of large upper ureteric stones as regard operative time, blood loss, hospital stay, post-operative pain, use of analgesia, intraoperative \& postoperative complications and success rate. Conclusion: LU provides a higher stone-free rate than URS in the management of large proximal ureteral stones. There are no differences regarding overall post-operative complications or major postoperative complications between the procedures. Semirigid URS is associated with a short operative time and length of hospital stay; however it leads to a higher need for auxiliary procedures. When counseling a patient with a large proximal ureteral stone, LU should be advised as the procedure with the higher chance of stone removal, although it is also more invasive, leading to longer operative time and length of hospital stay. Utilization of flexible ureteroscopy in conjunction with semi-rigid ureteroscopy may impact these outcomes, and deserves further systematic evaluation.
\end{abstract}

Keywords: Laparoscopic Ureterolithotomy, Ureteroscopy Management, Ureteral Stone

\section{Introduction}

There are multiple approaches for treatment of patients with upper ureteral stones. Extracorporeal shockwave lithotripsy (SWL), ureterorenoscopy (URS), percutaneous nephrolithotomy (PCNL), laparoscopic ureterolithotomy (LU) and open ureterolithotomy each has advantages and disadvantages. Although SWL is minimally invasive and can be performed as an outpatient procedure, disadvantages include a high retreatment rate, long treatment time, and inability to dissect a large or impacted stone (1).

URS is recommended as first-line treatment for upper ureteral stones. Flexible ureteroscopy with laser lithotripsy has a high surgical success rate, but this procedure necessitates special devices that are not available in all hospitals. PCNL is considered mainly for patients with a large stone burden and proximal ureteral stones $(2)$.

Laparoscopic ureterolithotomy can be used after first-line treatment has failed or is expected to fail. This approach may be a worthwhile alternative to open surgery (3), because the role of open ureterolithotomy declined rapidly after advancements of minimally invasive surgery ${ }^{(4)}$.

Laparoscopic surgery has been used for many types of urologic surgery involving ureterolithotomy in particular; the retroperitoneal approach has become established in laparoscopy since Geau developed a balloon dissection technique for the retroperitoneal space. Retroperitoneal laparoscopic ureterolithotomy is a new option minimally invasive technique to treat large, hard, long standing, and impacted upper ureter calculi in selected patients. However, large 
proximal ureteral stones are a challenge to minimally invasive techniques and management of stones larger than $15 \mathrm{~mm}$ is yet to be defined ${ }^{(\mathbf{5})}$.

\section{Aim of the Work}

The aim of this study is to compare between laparoscopic ureterolithotomy and retrograde ureteroscopy for treatment of large upper ureteric stones as regard operative time, blood loss, hospital stay, post-operative pain, use of analgesia, intraoperative \& postoperative complications and success rate.

\section{Ureteral Anatomy}

\section{Anatomical Considerations in The Ureteric Surgery}

The right and left ureters are retroperitoneal muscular tubes which originate from the renal pelvis and transport urine to the bladder in peristaltic waves. The length of the ureter varies with body habitus from 22 to 30 $\mathrm{cm}^{(6)}$

The ureter can be divided into three parts: proximal, middle, and lower. The proximal (Upper or lumbar) segment is from the ureteropelvic junction to the upper border of the sacrum; the middle (iliac) segment extends to the lower border of the sacrum which roughly corresponds with the iliac vessels; and the distal (lower or pelvic) segment extends from the sacrum to the bladder ${ }^{(6)}$, Figure 1.

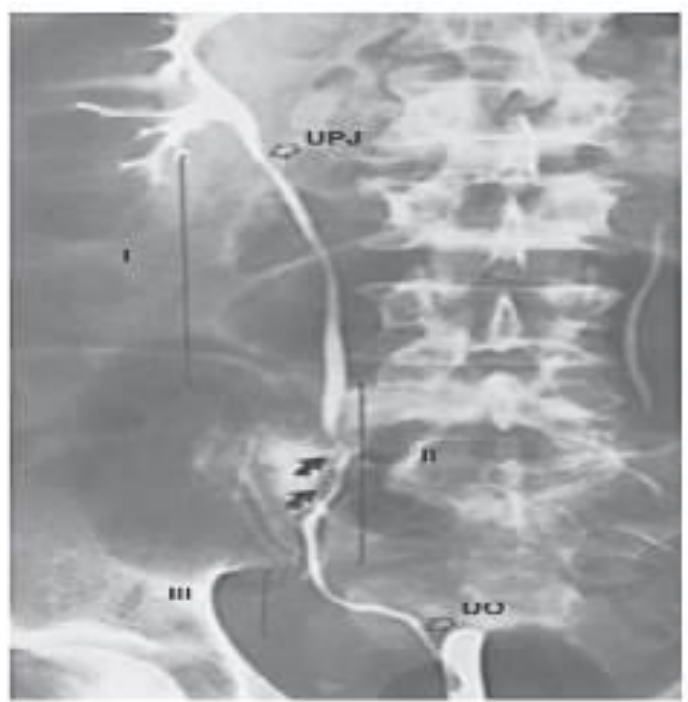

Figure 1: The right ureter, illustrated by retrograde injection of contrast material ${ }^{(6)}$.

\section{Normal variations in ureteral caliber:}

The ureter has not a uniform caliber, but it has 4 distinct narrowing's normally present along its course where the stones tend to lodge. They are at the urereropelvic junction, the pelvic brim as the ureter courses over the iliac vessels, the ureterovesical junction, and the ureteric orifice. The ureter is narrowest at the ureterovesical junction and as it traverses the intramural tunnel through the bladder wall. The intravesical course of the ureter measures about $0.5-1 \mathrm{~cm}$ with a diameter of 3-4 $\mathrm{mm}^{(6)}$ , Figure 2.

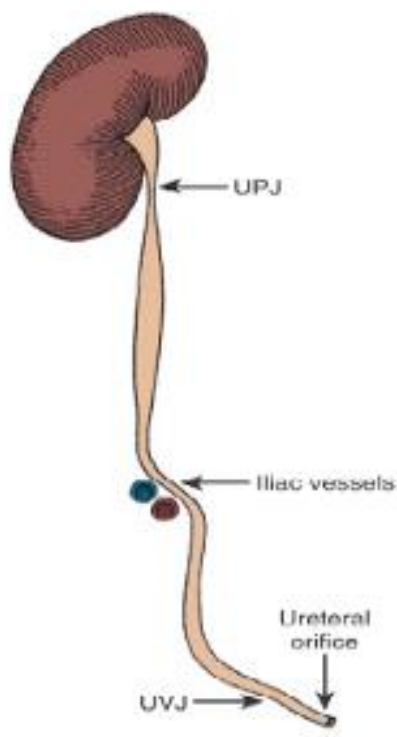

Figure 2: Sites of the narrowing along the course of the ureter ${ }^{(6)}$.

\section{Laparoscopic Anatomy}

\section{Introduction}

A clear and confident understanding of surgical, laparoscopic and applied anatomy of the kidney and ureter is a prerequisite for performing a percutaneous or a laparoscopic renal surgery ${ }^{(7)}$.

\section{Transperitoneal Laparoscopic anatomy:}

\section{Right ureter:}

The right kidney and ureter, when viewed in the transperitoneal approach, lies 
posterior to the ascending colon, inferior to the liver, and anterior to the psoas muscle. The first step in any approach to the right ureter is mobilizing the ascending colon and the white line of Toldt off Gerot's fascia ${ }^{(7)}$.

Laparoscopically, the defined layed of Gerota's fascia is clearly separate and evident from the mesenteric fat of the right mesocolon. The lateral border of the second portion of the duodenum may be attached to Gerota's fascia. When the duodenum is released from Gerota's fascia sharply (avoiding cautery so as to avoid thermal injury to the duodenum), the inferior vena cava (IVC) comes into view. The gonadal vein and ureter lie directly on the psoas muscle. The gonadal vein is encountered more medially and posteriorly than the ureter in most cases. One must also be aware that a relatively common venous abnormality for the right gonadal vein is to drain into the right renal vein, rather than the more usual ${ }^{(7)}$.

The renal vein is seen anterior to the pulsations of the renal artery. With the 30 degree laparoscope rotated to the 9 o'clock position, the posterior relationship of the renal artery to the renal vein is well appreciated. One must remember that the gonadal vein can insert into right renal vein, though its typical insertion is to the IVC. This is important as one may confuse the renal vein for the IVC ${ }^{(7)}$.

\section{Left ureter:}

The left kidney and ureter lies behind the descending colon, anterior to the psoas muscle and caudal to the spleen. The spleen lies directly adjacent to the superior pole of the kidney and should be mobilized away from the kidney early in the dissection to avoid splenic injury. The renal vein is seen lying anterior to the superior artery. Although not commonly seen in most laparoscopic case, the superior mesenteric artery, which courses directly anterior of the medial-most portion of the left renal vein, must always be kept in mind. The left gonadal vein drains into the left renal vein. The junction of the adrenal vein with the left renal vein is almost always medial to the gonadal vein insertion. A lumbar vein is frequently encountered on the posterior aspect of the left renal vein, which is far less common on the right renal vein. The lumbar vein location relative to the gonadal vein is more variable. The lumbar vein is best seen with gentle anterior traction on a clipped and ligated left gonadal vein. It is a good idea to have a nasogastric tube placed to keep the stomach decompressed. Although uncommon, the body of the stomach at times can lie just cranial to the upper pole of the left kidney. As stated above, the lumbar vein runs directly posterior from the posterior surface of the left renal vein. When specific identification of this vein is required as during a laparoscopic donor nephrectomy it is good to place a clip on the gonadal vein, leaving approximately a $2 \mathrm{~cm}$ stump attached to the renal vein. This can serve as a handle to obtain anterior traction on the renal vein, revealing the posterior lumbar vein (and potentially its branches) ${ }^{(7)}$

\section{Pathological Consequences of Impacted Ureteral Calculi}

\section{1 - Obstruction:}

There are many functional changes in the kidney associated with obstructive uropathy that affect renal hemodynamic variables and glomerular filtration. These are influenced by the extent and severity of obstruction, whether obstruction is unilateral or bilateral, and whether the obstruction currently persists or has been relived ${ }^{(8)}$.

Ureteral obstruction, whether partial or complete, produces a progressive decrease in renal excretory function. After obstruction, a rapid redistribution of renal blood flow from medullary to cortical nephrons occurs. This redistribution results in a decrease in glomerular filtration rate (GFR) and renal plasma flow, reflecting a decrease in both glomerular and tubular function ${ }^{(\mathbf{9})}$.

\section{A) Renal response to complete unilateral ureteral obstruction (UUO):}

During the first 1 to 2 hours, ipsilateral renal flow and ureteral pressure rise. In a second phase lasting 3 to 4 hours, renal blood flow begins to decline, while ureteral pressure continue to rise. In third phase beginning about 5 hours after the obstruction, both renal blood flow and ureteral pressure fall (10). 
Both prostaglandin E2 (PGE2) and nitrous oxide (NO) contribute to the net renal vasodilatation that occurs early following UUO. Infusion of angiotensin-converting enzyme (ACE) inhibitor captopril attenuates the declines in renal blood flow (RBF) and GFR in UUO, suggesting that angiotensin II is an important mediator of the preglomerular vasoconstriction occurring during the second and third phases of UUO ${ }^{(\mathbf{1 1})}$.

In contrast to the early powerful renal vasodilatation with UUO, there is a modest increase in $\mathrm{RBF}$ with bilateral ureteral obstruction (BUO) lasting for about 90 minutes followed by a prolonged and profound decrease in RBF that is greater than that found with UUO. The shift seen with UUO of blood flow from outer to inner cortex is the opposite with BUO. This difference in the pathophysiology between UUO and BUO is due to accumulation of vasoactive substances e.g. atrial natruretic peptide (ANP) in BUO that causes preglomerular vasodilatation and postglomerular vasodilatation ${ }^{(\mathbf{1 1})}$.

\section{B) Ureteric Changes with Calcular Obstruction:}

Obstruction results not only in decreased renal function but also fairly rapid changes in the ureteral peristaltic. There is hypertrophy of ureteral musculature after only 3 days of obstruction. If obstruction continued for 2 weeks, connective tissue deposits (scar) occur between muscle bundles. Such changes are considered marked at eight weeks. Chronic ureteral obstruction results in decreased peristalsis and decreased pressure. The presence of urinary infection totally impairs the ureteral function. Perhaps this is why fewer stones pass spontaneously in patients with infection. Doppler ultrasound may be used to evaluate normal ureteric peristalsis by showing periodic jetting of urine flow into the urinary bladder lumen from the ureteric orifice. In patients with acute renal colic, Doppler studies may show absence of urine flow as compared with the opposite normal side or, in patients with partial obstruction, relatively continuous low velocity urine flow contrasted to the periodic peristaltic jet of the normal side ${ }^{\mathbf{( 1 2})}$.

\section{Management of upper ureteric stones}

\section{Diagnosis of upper ureteric stones:}

Diagnosis of upper ureteric stones is made on the basis of information obtained from the history, physical examination, laboratory investigations, and radiographic studies ${ }^{(\mathbf{1 3})}$.

\section{Symptoms and Signs of upper ureteric Stone disease:}

Patients with upper ureteric stones classically present with unilateral flank pain of sudden onset and progressive course. The pain is precipitated by passage of a renal stone from the renal pelvis to the ureter, and is due to impacted upper ureteric stone causing ureteral spasm. The pain is often severe enough to prompt the patient to seek medical care at an emergency department, and can be accompanied by nausea and vomiting. Pain is located in the lumber region of that side and radiates anteriorly to the abdomen and to the groin and external genitalia of the same side. Less commonly, it can be manifest as microscopic or gross hematuria without pain. Signs and symptoms of sepsis as fever, tachycardia can occur in case of obstruction with infection ${ }^{(13)}$.

\section{Physical examination:}

The patient with upper ureteric stone may be in obvious pain and may constantly adjust position to alleviate the discomfort. Ipsilateral costovertebral angle tenderness may be present. Comorbid diseases should be identified, particularly any systemic illnesses that might increase the risk of stone formation or that might influence the clinical course of the disease, other personal or family history of stone passer, with previous treatment and stone analysis, and any anatomical abnormalities or surgical interference of the urinary tract, complete history of drugs use can help identify those known to increase the risk of stone formation ${ }^{(13)}$.

\section{Risk groups for stone formation:}

The risk status of a stone former is of particular interest as it defines both probability of 
recurrence or regrowth of stones and imperative for pharmacological treatment. About $50 \%$ of all recurrent stone formers have just one recurrence during lifetime. High recurrence rate is slightly more than $10 \%$ of all stone formers. Chemical composition of the stone and past history of stone passer indicates the high recurrence rate for stone recurrences ${ }^{(\mathbf{1 4})}$.

\section{Laboratory Investigations:}

\section{Laboratory Investigations typically carried out include:}

\section{Microscopic examination and culture of the urine:}

Microscopic examination of urine sample which may show red blood cells, bacteria, leucocytes, urinary casts, and crystals. Urine culture is important to identify any infecting organisms that may be present in the urinary tract and sensitivity to determine the susceptibility of these organisms to specific antibiotics ${ }^{(13)}$.

\section{Complete blood count (CBC) and renal function tests:}

Complete blood picture may indicate high total leucocytic count which is suggestive of bacterial infection, as seen in the setting of struvite stones. Renal function tests to look for blood urea, serum creatinine which is a direct reflection of renal function, serum uric acid, abnormally high total blood calcium levels ${ }^{(\mathbf{1 5})}$.

\section{Radiological investigations:}

Plain $X$ ray of the abdomen and pelvis (kidney, Ureter, Bladder KUB).

Abdominal plain $\mathrm{x}$ ray is a very simple investigation. It is available at nearly all hospitals. It is economical, high skill is not required to perform this investigation, and it can be interpreted easily. The disadvantages are radiation hazard that cannot be performed during pregnancy. The picture may not be satisfactory in the presence of gas and fecal matter in the intestine (not well prepared). Radio opaque shadows in the area of ureter may be seen. Only non radio- opaque (radio lucent) stones are not seen and require contrast medium radiological investigations for confirmation of the diagnosis. However, a KUB can be helpful in differentiating between radiolucent and radiopaque stones and during follow up ${ }^{(16)}$.

\section{Pelvi- abdominal ultrasound:}

Ultrasonography (Us) should be used the primary procedure. It is a safe (no risk of radiation), reproducible, and inexpensive method of urinary stone detection. Currently, US is recommended in patients in whom radiation exposure is a concern, such as pregnant or pediatric patients. Ultrasonography can identify stones located in the renal calices, renal pelvis, pelvi-ureteric junction, and vesicoureteric junction, as well as dilatation of the upper urinary tract. For renal stones $>5 \mathrm{~mm}$, ultrasound has a sensitivity of $96 \%$ and a specificity of nearly $100 \%$. For all stone location, the sensitivity and specificity of ultrasound reduces to $78 \%$ and $31 \%$ respectively. When urinary tract calculi cause obstruction, US is very effective in demonstrating the secondary sign of hydronephrosis ${ }^{(\mathbf{1 7})}$.

\section{Computed tomography:}

Non-contrast enhanced CT (NCCT) abdomen and pelvis, has become the standard method for diagnosing upper ureteric stones. It has replaced intravenous urography (IVU), which was previously the gold standard for many years. It can also identify the presence of the stones, its diameter, and density. NCCT show the higher sensitivity and specificity in identifying urinary stones. Uric acid and xanthine stones are radiolucent on plain $\mathrm{x}$ ray films and can be detected by NCCT. However, indinavir stones cannot be detected on NCCT. In addition, NCCT can determine mean stone density and skin-to-stone distance, both of which affect ESWL outcome. However, the advantage of a non-contrast imaging modality must be balanced against the loss of information on renal function and the anatomy of the urinary collection system, as well as the higher radiation dose of NCCT ${ }^{(18)}$, Figure 3. 


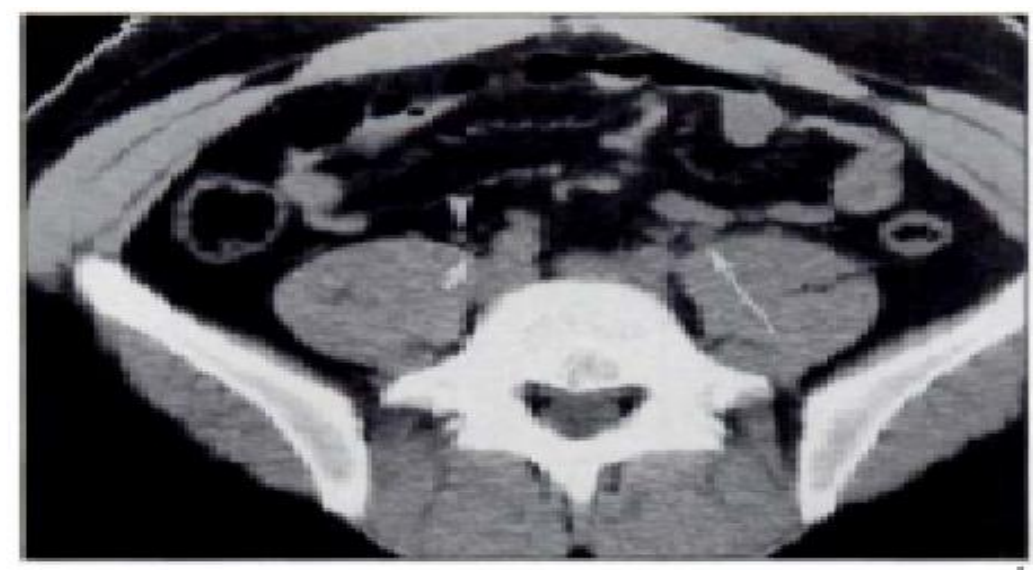

Fig. 3: CT coronal section of LT ureteric dilatation compared to normal RT ureter ${ }^{(19)}$.

\section{Treatment of upper ureteric stones}

\section{A. Conservative Management}

\section{Indications and patient selection}

In order to select appropriate therapy, the stone suitable for conservative therapy should be determined, and also the time needed before stone passage, and factors predictive of spontaneous passage are important to be determined. Immediate intervention for ureteric calculi is absolutely warranted in the presence of urinary infection, complete ureteric obstruction (bilateral ureteric stones or obstructed solitary kidney), significant symptoms prompting multiple office visits, or for patients with occupational requirements precluding conservative treatment e.g. pilots, physicians, and astronauts ${ }^{(20)}$.

\section{Duration of conservative management}

The time interval of spontaneous passage is important to minimize the pain expectations and the potential risk of renal injury as possible. Together, stone size, location and side were related to stone passage interval. The average interval to stone passage was $1-2$ weeks for stones less than $5 \mathrm{~mm}$. A common reason for intervention is poor pain control followed by non progression on serial imaging after an adequate period of observation. Patient considering observation need to allow from 2 to 4 weeks in order to give an adequate trial ${ }^{(\mathbf{2 1})}$.

\section{Lines of medical management ${ }^{(21)}$}

\section{a. Pain and Nausea Treatment.}

\section{b. Alpha Blockers.}

\section{c. Oral Steroids, Calcium Channel Blockers, and Beta Blockers:}

\section{d. Other miscellaneous Agents Studied in Conservative Stone Management.}

\section{e. High Water Intake.}

\section{B - Interventional Treatment:}

Indications of urological interference in impacted upper ureteric stones:

The stones which fail to come out after conservative treatment should be removed endoscopically or surgically. According to the AUA guideline 2009, the prerequisites for conservative treatment of ureteric stones are a stone size $<10 \mathrm{~mm}$, well controlled pain, no clinical evidence of sepsis and adequate renal functional reserve. Regular imaging should be performed to monitor stone progression and to assess upper tract obstruction. Stone removal is indicated in stones $>10 \mathrm{~mm}$ and in stones when there is persistent obstruction, uncontrolled pain or sepsis. Upper ureteric stones can be treared by Ureteroscopy through both retrograde approach and Antegrade approach. Despite the versatility of modern URS, definitive treatment of urinary stones remains the most common indication for performing 
ureteroscopic techniques especially when the conservative approach to ureteric stones in contraindicated or fails ${ }^{(22)}$.

\section{Instruments and equipments}

a. Guidewires.

b. Dilation devices.

c. Semirigid ureteroscopes \& nephroscopes.

d. Flexible ureteroscopes.

e. Lithotripsy devices.

f. Stones extraction devices.

g. Ureteric occlusion devices ${ }^{(23)}$.

\section{Technical consideration of retrograde ureteroscopy}

\section{Patient preparation :}

Before URS, anticoagulants should be stopped 7-10 days before the procedure and if necessary to shift to low-molecular-weight heparin at the evening of operation, Also treatment of urinary infection should be considered ${ }^{(24)}$.

\section{Patient positioning and anaesthesia:}
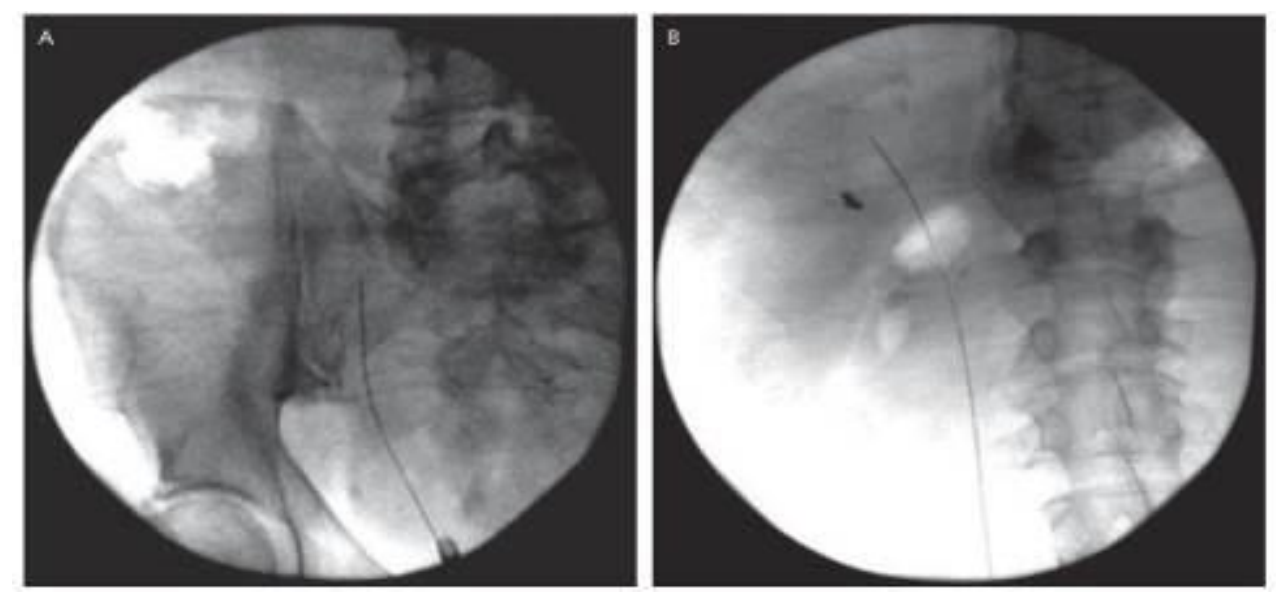

Fig. 4: Guidewire advanced to (A) mid ureter and (B) level of kidney ${ }^{(24)}$.

c. Visualization of the radio-opaque stone under fluoroscopy: When the stone is not visualized, retrograde ureterography may be used ${ }^{(23)}$.
Patient is placed in lithotomy position under spinal anaesthesia which has been demonstrated to be safe and feasible for URS. However, the general anesthesia is preferred for proximal ureteric stones especially with prolonged procedures ${ }^{(24)}$.

\section{Ureteric Access:}

a. Identification of the ureteric orifice . If identification is difficult, methylene blue or indigo carmine may be injected.

b. Insertion of a guidewire into ureteric orifice under guidance of a ureteric catheter and fluoroscopy (Fig. 4). Then, it may be helpful to place a second guidewire to serve as a safety wire after URS insertion ${ }^{(24)}$.

During the procedure, care should be taken to avoid ureteric injury, such as submucosal passage or ureteric perforation, especially with kinked ureter or when the ureter is inflamed and edematous at the level of stone impaction. Also care should be taken to avoid retrograde propulsion of the stone during guidewire insertion. Flexible ureteroscopy can be inserted directly into the ureter over the guidewire ${ }^{(24)}$. 
ureteric wasting. It should not be inflated to a pressure greater than recommended by the manufacturer ${ }^{(23)}$.

\section{Lithotripsy and stone extraction:}

In many cases, the treatment of ureteric calculi will require lithotripsy. Then, remaining fragments can be either extracted utilizing previously described extraction devices, or allowed to pass with or without the aid of a ureteric stent. Ureteroscopic removal of small ureteric stones with a basket is a relatively quick procedure with a lower morbidity rate than lithotripsy. The basket technique should be attempted only for small distal ureteric calculi for the proximal ones. Small ureteric stones or fragments can be removed fast and safely with forceps which can be better controlled than a basket ${ }^{(23)}$.

\section{Post- ureteroscopy stenting:}

\section{1) Indications of post- ureteroscopy stenting:}

Routine stenting after uncomplicated URS is optional and may not be necessary. A ureteric catheter may be inserted for 1 or 2 days to decrease postoperative pain and stricture formation. Ureteric stenting is indicated after the completing of URS either by a ureteric catheter for 2-3 days or a doubleJ stent for 2-4 weeks. These include ureteric injury or severe laceration or perforation at the site of impaction, stricture, renal insufficiency, solitary kidney, after bilateral URS, or if there is significant residual stone burden ${ }^{(25)}$.

\section{2) Factors against post- ureteroscopy stenting:}

Post-ureteroscopy stenting may be disadvantageous owing to development of postoperative pain, narcotic use, and lower urinary tract symptoms more than in non stented patient. Also additional cost may be added by stenting following uncomplicated ureteroscopic procedures ${ }^{(7)}$.

\section{Results of ureteroscopic management Results of URS:}

Generally; URS treatment appears to decrease the time interval required to become stone-free after treatment. In a report by Elashry and his colleagues, the introduction of the 7.5 Fr flexible URS was reported to significantly decrease the need for dilation of the ureteric orifice, postoperative analgesia requirement, and need for postoperative hospitalization. For proximal ureteric calculi; Ho: YAG LASER lithotripsy is highly efficient irrespective of stone type or burden with stone-free rate of $97 \%$. Beside Ho: YAG LASER, favorable fragmentation and stonefree rates have been reported using other forms of intracorporeal lithotripsy including EHL and pneumatic lithotripsy ${ }^{(26)}$.

\section{Technical aspects of Retroperitoneal Laparoscopic Ureterolithotomy "RPLU"(27)}

Gill and his colleagues ${ }^{(27)}$ identified that the access technique for retroperitoneoscopy differs from transperitoneal laparoscopic access in three key aspects:

1) Location and technique of primary trocar placement.

2) Optimal positioning of the balloon dilator.

3) Technique for safe placement of secondary ports.

Gill and coworkers ${ }^{(27)}$ presented an effective technique to obtain an access for retroperitoneoscopic proximal ureteric surgery achieving the following (27).

1) Minimizes carbon dioxide leak and subcutaneous emphysema.

2) Places he balloon dilator within Gerot's fascia with visualization of the anterior and posterior renal surfaces.

3) Allows optimal and safe placement of secondary ports, and minimizes peritoneal transgression.

\section{Technical aspects of Transperitoneal Laparoscopic (TPLU): Ureterolithotomy}

\section{a. Patient positioning:}


The patient is placed in the $45^{\circ}$ to $60^{\circ}$. Flank position with the kidney-bridge elevated
(Fig. 5). The surgeon and the assistant stand facing the front of the patient ${ }^{(28)}$.

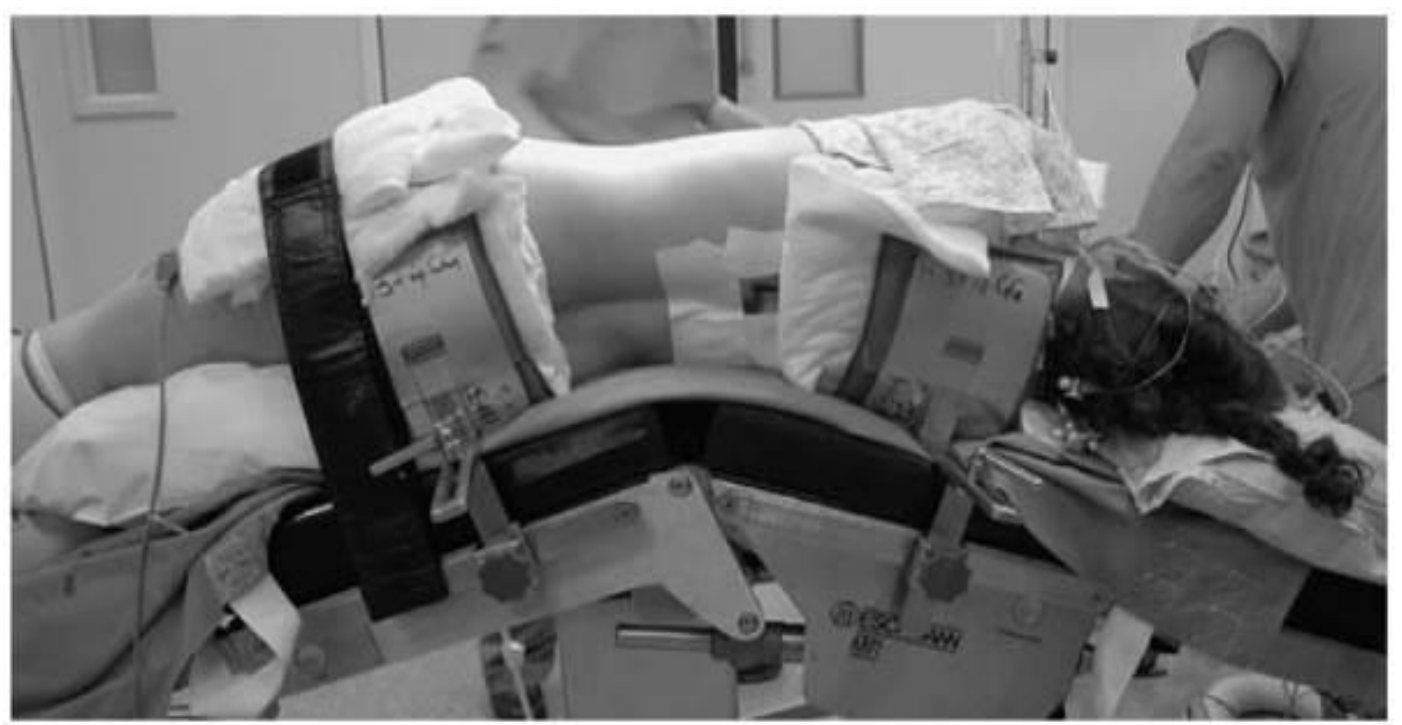

Fig. 5: Patient positioning in TPLU ${ }^{(29)}$.

\section{Pneumoperitoneum and port placement:}

Peritoneal access in gained using a Veress needle. The abdomen is insufflated with carbon dioxide to a pressure of 15 $\mathrm{mmHg}$. The first port $(10 \mathrm{~mm})$ is placed mid way between the umbilicus and the anterior superior iliac spin, at the lateral edge of the rectus muscle. The second port $(5 \mathrm{~mm})$ in placed at the angle between the costal margin and the lateral border of the ipsilateral rectus muscle. The third port $(10$ or $12 \mathrm{~mm})$ is placed between these two ports. This port may alternatively be placed in the umbilicus ${ }^{(28)}$.

\section{Mobilization of the colon and identification of the ureter:}

Mobilization of the colon is performed by incising along the line of Toldt, up to the liver on the right and the splenic ligaments on the left. This allows the colon, spleen, and pancreas to be reflected away from the left kidney (Fig. 6). Once the colon has been mobilized, the ureter is identified above the psoas muscle (Fig. 7). The procedure is then carried out in the same fashion of the retroperitoneal approach ${ }^{(28)}$.

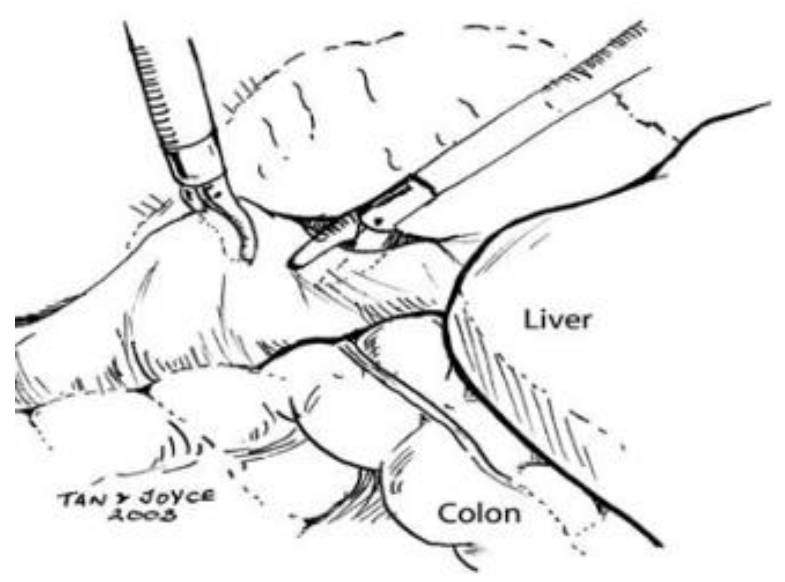

Fig. 6: Colon mobilization ${ }^{(29)}$. 


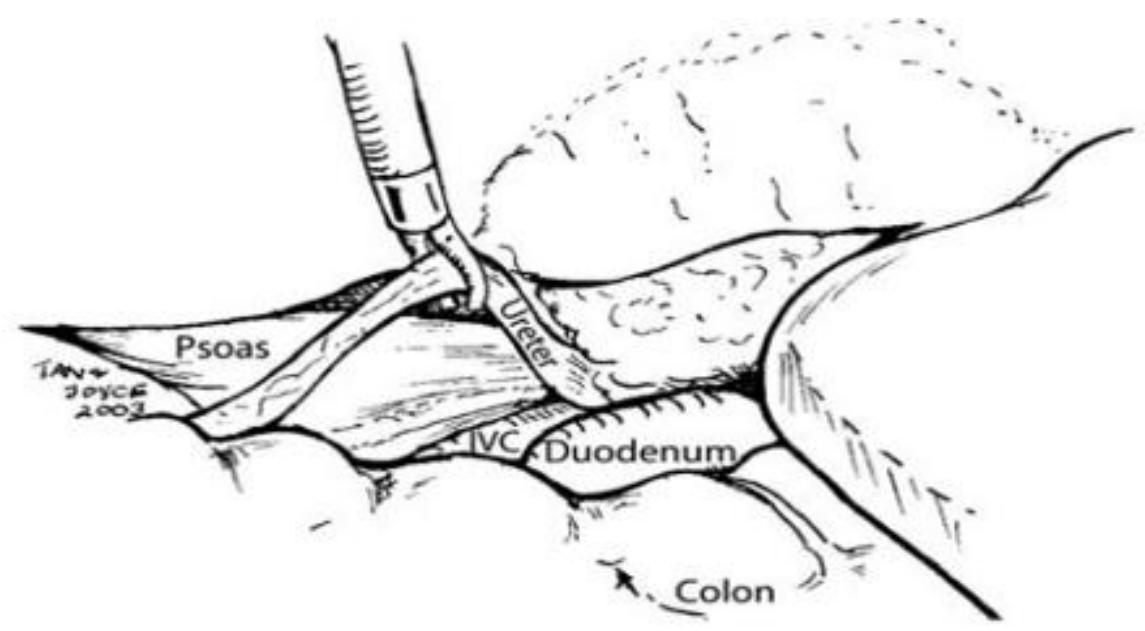

Fig. 7: Identification of the ureter, gonadal vein and psoas muscle "key landmark" (29).

\section{Complications of laparoscopic ureterolithotomy}

\section{a. Intra operative complications:}

Operative complications may be recognized immediately, or may be identified later. Several reports have addressed specific complications. Conversion to open surgery was reported in a many series; in some of them it was related to the failure to locate the ureter because of severe adhesions or due to major vascular injury. Most of the cases converted to open surgery were during the initial learing curve. Most of the vascular injuries recorded were nearly of the same incidence like other urologic laparoscopic procedures. The commonest injured vessels were the inferior vena cava, and to lesser extent the gonadal and lumbar veins ${ }^{(30)}$.

Also conversion from retroperitoneal to transperitoneal approach was recorded due to the limited working place during RPLU. Conversion may result also from technical faults in the procedure as in case of poor pneumoretroperitoneum or accidental ureteric avulsion ${ }^{(31)}$.

Unrecognized peritoneal injury during RPLU may occur with secondary port placement with subsequent injury to the intraperitoneal structures. However the potential for injury of these structures during RPLU is minimal compared to TPLU. Other laparoscopic access injuries including visceral or bowel injuries or injury of abdominal wall vessel are rarely reported with the procedure (27).

\section{b. Postoperative complications:}

Jeong and coworkers ${ }^{(9)}$ evaluated the role of RPLU for proximal ureteric stones in 12 patients. Except for one patient with delayed urinary leakage (8\%), these investigators had no serious postoperative complications. This leakage can lead to subsequent urinoma or haematoma. However this collection will be of better prognosis if following RPLU than TPLU; because in RPLU this collection will be confined only to the retroperitoneal space. In such cases, the tube drain should be kept for longer period postoperatively with fixation of a ureteric stent (if not fixed before) for better drainage of the collection and giving a chance for good healing of the ureter.

Urinary after is also related to the surgical technique in closure of the ureterotomy after stone extraction. In some series in which the ureter was left open in a number of case $(31 \%)$, the mean period of post-operative urinary leakage was prolonged up to 5.5 days. This period was reduced to 3.2 days by stenting and suturing of the ureter. Cutting of the ureter with endoknife or diathermy made no difference in the incidence of leakage. The main predisposing factors for the incidence of leakage were the chronic 
inflammation, oedema and friability of the ureter especially with prolonged impaction and presence of infection ${ }^{(32)}$.

\section{Problems anticipated and preventive measures taken during laparoscopic ureterolithotomy for upper ureteral stones:}

Injury to the subcostal vessels is prevented by keeping a space of 1-1.5 cm below the tip of the $12^{\text {th }}$ rib. Opening up separating the peritoneum from the abdominal wall muscle by gentle finger dissection carried out anteriorly for 2-3inches, after the reroperitoneal fat is seen through the split muscles. Identification of the ureter is done by observing the peristalsis and the typical blood vessels of the ureter. Structures like the gonadal vein and the inferior vena cava that can be mistaken for the ureter should be kept in mind. Upward migration of the stone is prevented by placing a Babcock type forceps on the ureter above the stone bulge ${ }^{(\mathbf{3 3})}$.

\section{Conclusion}

- Although different treatment modalities have been used for large impacted ureteral stones, the optimal treatment for these stones remains controversial.

- LU provides a higher stone-free rate than URS in the management of large proximal ureteral stones. There are no differences regarding overall postoperative complications or major postoperative complications between the procedures. Semi-rigid URS is associated with a short operative time and length of hospital stay, however it leads to a higher need for auxiliary procedures. When counseling a patient with a large proximal ureteral stone, LU should be advised as the procedure with the higher chance of stone removal, although it is also more invasive, leading to longer operative time and length of hospital stay. Utilization of flexible ureteroscopy in conjunction with semi-rigid ureteroscopy may impact these outcomes, and deserves further systematic evaluation.

\section{References}

1. Kreshover JE, Diskstein RJ, Rowe C et al. (2011): Predictors for negative ureteroscopy in the management of upper urinary tract stone disease. Urology, 78: 748- 752.

2. Yencilek F, Sarica K, Erturhan S et al. (2010): Treatment of ureteral caculi with semirigid ureteroscopy: Where should we stop? Urol. Int., 84: 260-264.

3. Leonardo $\mathrm{C}$, Simone $\mathrm{G}$, Rocco $\mathrm{P}$ et al. (2011): Laparoscopic Ureterolithotomy: Minimally invasive second line treatment. Int. Urol. Nephrol., 43: 651654.

4. Lee $\mathrm{H}$, Ryan $\mathrm{T}$, Teichman $\mathrm{J}$ et al. (2003): Stone retropulsion during holmium: YAG lithotripsy. J. Urol., 169: 881-885.

5. Zhu H, Xiongjn $Y$, Xuren $X$ et al. (2014): Retrograde, Antegrade, and Laparoscopic Approach to the Management of Large Upper Ureteral Stones After Shockwave Lithotipsy Failure: A Four- Year Retrospective Study. J. Endourol., 28: 100-103.

6. Shnorhavorian M, Anderson K (2004): Anatomic and physiologic considerations in ureteroscopy. Urol. Clin. N. Am., 31: 15- 34.

7. Denstedt J, Wollin T, Sofer M et al. (2001): A prospective randomized controlled trial comparing non-stented versus stented ureteroscopic lithotripsy. J. Urol., 165: 1419-1422.

8. Alan J, Wein MD, Louis $\mathrm{R}$ et al. (2007): Pathophysiology of Urnary Tract Obstruction. In Walsh PC, R.C Campbell's Urology. Vol 2. $9^{\text {th }}$ ed. Philadelphia, Pa: WB Saunders, 1195 26.

9. Jeong BC, Park HK, Byeon SS et al. (2006): Retroperitoneal Laparoscopic Ureterolithotomy for upper ureter stones. J. Korean. Med. Sci., 21 (3): 441-444.

10. Burge $H$, Middleton W, McClennan B et al. (1991): Ureteral jets in healthy 
subjects and in patients with unilateral ureteral calculi: Comparison with color Doppler US. Radiology, 180: 437-488.

11. Misseri R, Rink RC, Meldrum DR et al. (2004): Inflammatory mediators and growth factors in obstructive renal. J. Surg. Res., 119: 149- 159.

12. Gee VVF, Kiviat MD (1985): Ureteral response to partial obstruction: Smooth muscle hyperplasia and connective tissue proliferation. Invest. Urol., 12: 309-316.

13. Anoia EJ, Paik ML and Resnick MI (2009): Anatrophic Nephrolithomy, in Graham and Keane. pp. 45-50. https://infogalactic.com/info/Kidney_sto ne

14. Straub M, Strohmaier WL, Berg W et al. (2005): Diagnosis and metaphylaxis of stone disease Consensus concept of the National Working Committee on Stones Disease for the Upcoming German Urolithiasis Guideline. World J. Urol., 23(5): $309-23$.

15. Pietrow PK, Karellas ME (2006): Medical Management of Common Urinary Caculi. Amercican Family Physician, 74(1):86- 94.

16. Kennish SJ, Bhatnagar P, Wah TM et al. (2008): Is the KUB radiograph redundant for investigating acute ureteric colic in the non-contrast enhanced computed tomography era? Clin. Radiol., 63(10): 1131-1135.

17. Varma G, Nair N, Salim A et al. (2009): Investigations for recognizing urinary stone. Urol. Res., 37 (6):349352.

18. Patel T, Kozakowski K, Hruby G et al. (2009): Skin to stone distance is an independent predictor of stone-free status following shockwave lithotripsy. J. Endourol., 23(9): 1393- 1395.

19. Smith RC, Verga M, Dalrymple NC et al. (1996): Acute ureteral obstruction: Value of secondary signs on helical unenhanced CT. AJR., 167: 1109- 1113.

20. Segura J, Preminger G, Assimos D et al. (1997): Ureteral Stones : Clinical Guidelines panel summary report on the management of ureteral caculi. The Amercican Urological Association $\mathrm{J}$. Urol., 158: 1915- 1921.

21. Miller O, Kane C (1999): Time to stone passage for observed ureteral caculi: a guide for patient education. $\mathrm{J}$. Urol., 162: 688- 691.

22. Honeck P, Wendt-Nordahl G, Krombach P et al. (2009): Does open stone surgery still a role in the treatment of urolithiasis? Data of a primary urolithiasis center. J. Endourol., 23(7): 1209-1212.

23. Rapp D, Gerber G (2006): Ureteroscopy. In Advanced Endourology: The Complete Clinical Guide. Edited by: S. Nakada and M. Pearle (eds.). Humana Press Inc., Totowa, New Jersey. Chapter 6, pp.87104.

24. Knoll T, Michel M (2005): Ureterorenoscopy. In: Manual Endourology. R. Hohenfellner, and J. Stolzenburg (eds.). Springer Medizin Verlage Heidelberg, Germany. Chapter 12, pp. 105-115.

25. Cheung $M$, Lee $\mathrm{F}$, Leung $\mathrm{Y}$ et al. (2003): A prospective randomized controlled trial on ureteral stenting after ureteroscopic holmium laser lithotripsy. J. Urol., 169: 1257-1260.

26. Sofer M, Watterson J, Wollin $\mathbf{T}$ et al. (2002): Holmium: YAG laser lithotripsy for upper urinary tract caculi in 598 patients. J. Urol., 167:31-34.

27. Gill I, Grune $M$ and Munch L (1996): Access technique for retroperitoneoscopy. J. Urol., 156: 1120 -1124 .

28. Stifelman M (2003): Operating Room Set-Up and Accessing the Abdomen. In Essential Urologic Laparoscopy: The Complate Clinical Guide. S. Nakada (eds.). Humana Press Inc., Totowa, New Jersey, Usy.,Chaper 3, pp. 23-35.

29. Tan A, Joyce A (2005): Transperitoneal laparoscopic readical nephrectomy. In Laparoscopic urologic surgery in malignancies. J. De la 
Rosette, and I. Gill (eds.). Springer, Berlin., Chapter 2.1, PP. 19-28.

30. Jones DA, Atherton JC, O'Reilly PH et al. (1989): Assessment of the nephron segments involved in postobstructive diuresis in man, using lithhum clearance. Br. J. Urol., 64: 559563.

31. Hossain S, Hossain A, Khan $S$ et al. (2006): Retroperitoneoscopy for treatment of ureterl stones. Urology Supplement, 5: 177-190.

32. Gaur D, Trivedi S, Prabhudesai M et al. (2002): Laparoscopic ureterolithotomy: technical considerations and long-term followuop. BJU. Int., 89: 339-343.

33. Van der Vort M, Heijnsdijk EAM and Gouma DJ (2004): Bowel injury as a complication of laparoscopy. Brit. J. Surg., 91: 1253-1258. 